Paper ID \#20000

\title{
The Quantitative Microbial Risk Assessment Interdisciplinary Instructional Institute (QMRAIII) - A Platform for Cross Disciplinary Training of Engi- neers with Social and Biological Scientists to Address Public Health Issues
}

\author{
Dr. Jade Mitchell, Michigan Sate University, Dept. of Biosystems and Agricultural Engineering
}

Dr. Jade Mitchell is an Assistant Professor in the Department of Biosystems and Agricultural Engineering at Michigan State University. She received her B.S. from the University of Pittsburgh in Civil and Environmental Engineering, M.S. in Civil Engineering and Ph.D. in Environmental Engineering from Drexel University. Dr. Mitchell is keenly interested in supporting environmental and human health decision making through the use of integrated risk and decision frameworks. Her work includes development of new frameworks, models and data sets toward this end. Dr. Mitchell has specific experience in quantitative microbial risk assessment (QMRA) and she conducted her graduate research under the multi-University, multi-disciplinary, Center for Advancing Microbial Risk Assessment (CAMRA), a jointly funded US EPA and DHS Center of Excellence. After joining MSU, she became affiliated with CAMRA as a principal investigator. Her lab conducts both computational risk modeling research and fundamental research. Her current work and future interests lie at the intersection of chemical and microbial stressors where understanding trade-offs, benefits and risks deviate from existing risk paradigms and require new data, tools and frameworks. Her future research goals include applications of risk-based decision making to water infrastructure management, and emerging hazards such as antibiotic resistance. She is managing editor and a developer of the QMRAwiki, an interactive, online tool for the QMRA community. Dr. Mitchell has also been involved in developing and teaching training workshops in QMRA for several years. She was recently awarded a nearly $\$ 1 \mathrm{M}$ grant from the National Institutes of Health to develop a new course, models and tools to support interdisciplinary engagement in QMRA.

\section{Dr. Mark H. Weir, The Ohio State Universiry}

Mark H. Weir earned his BS in Environmental Engineering from Wilkes University and Ph.D. in Environmental Engineering from Drexel University. He worked as the Associate Director of the Center for Advancing Microbial Risk Assessment. He worked in risk research and engineering with the US EPA until leaving for a faculty position at Temple University. While at Temple he served as the Acting Division Director of the Environmental Health Division in the College of Public Health. Dr. Weir is now at The Ohio State University working with both the College of Public Health and College of Engineering. He specializes in predictive water quality and risk models constructed to be easy to use for operations workers and managers.

Prof. Julie Libarkin

Dr. Libarkin is a Professor of Geoscience Education at Michigan State University in the Department of Earth and Environment Sciences and CREATE for STEM Institute for Research on Science and Mathematics Education. Currently, her research focuses on cognition, assessment of student learning, validity and reliability in research, curriculum and visual design, and discipline-based education research.

Dr. Joan B. Rose

(c)American Society for Engineering Education, 2017 


\title{
The Quantitative Microbial Risk Assessment Interdisciplinary Instructional Institute (QMRAIII) - A Platform for Cross Disciplinary Training of Engineers with Social and Biological Scientists to Address Public Health Issue
}

\begin{abstract}
The recent and tragic water crisis in Flint, MI highlights the need for better linkages between engineers, public health agencies and public policy officials. Quantitative microbial risk assessment (QMRA) is an interdisciplinary field and research domain that addresses exposures to microbial pathogens through the environment. It is a widely accepted framework for the study of water quality and food safety. QMRA is most often performed by engineers because it is computationally intensive to mathematically model dynamic physical, chemical and biological processes from source to adverse health outcomes in a receptor. However, it relies heavily on multidisciplinary contributions for effective implementation. Risk management includes the design of engineering controls but also risk communication and risk perception - social science topics that are integral components of risk analyses. Furthermore, exposure to microbial agents is associated with substantial variability and uncertainty in behavior across the population. Despite the utility of the QMRA approach, few biological or social scientists are trained in this process or have sufficient statistical and quantitative skills to undertake such analyses. Similarly, few engineers possess the necessary skills in the social sciences to adequately address issues of human behavior that affect risks associated with exposure to pathogenic agents or responses to health risks. Therefore, we developed an intensive short course to enhance multidisciplinary cross training and graduate research through a combination of lectures and experiential learning opportunities - hands-on exercises and a group project. Through a comprehensive evaluation plan of the course conducted in 2015, we assessed the expanded ability of the course to improve outcomes for engineers in areas of the risk paradigm not traditionally emphasized in their graduate programs. Likewise, we tested the ability of the course to improve the quantitative and modeling skills in participants with backgrounds in social and behavioral sciences. The survey instruments were developed to evaluate both real and perceived knowledge of the participants. This paper focuses on our assessment of the extent to which perception was changed by learning. Participants completed identical pre- and post- workshop surveys containing five Perceptions scales (Perceptions of: QMRA-Related Ability, QMRA-Specific Ability, Knowledge - Hazard Identification, Knowledge - Dose Response and Exposure Assessment, Knowledge - Risk Management). This paper describes the structure of the course, content, pedagogy, and the lessons learned through the participant evaluation and perception of learning. Modifications to the course in response to participant comments were made between the 2015 and 2016 course and will be presented as well. Finally, this paper will serve as a model going forward for our subsequent short courses and may also be used as a model for cross disciplinary training of engineers within other domains that require social science integration.
\end{abstract}




\section{Introduction and Background}

Quantitative microbial risk assessment (QMRA) is globally accepted for addressing probabilities of disease using mathematical approaches and integrated data sets for human health threats associated with exposure to etiological agents in a diverse set of indoor and outdoor environments. QMRA is inherently interdisciplinary and requires some understanding of biological processes and methodology that focuses on dynamic and complex systems. Because few specialists in QMRA exist, risk assessments are generally conducted by scientists from multiple research disciplines as new needs arise. Most often QMRA is performed by engineers because it is computationally intensive to mathematically model dynamic physical, chemical and biological processes from source to adverse health outcomes in a receptor. Risk assessment provides a science based interface to policy and requires that the science is adequately communicated to diverse stakeholders. Few engineers possess the necessary skills in the social sciences to adequately address issues of human behavior that affect risks associated with exposure to pathogenic agents or responses to health risks. Quantitative information is particularly challenging to communicate, but can greatly improve decision and policy making.

QMRA methods have particular importance for mitigating risks from emerging or reemerging pathogens. It appears that emerging pathogen events are increasing, and that this trend cannot be fully explained by increases in surveillance or research (Jones et al., 2008). Additionally, climate change, land use change, and transportation networks have altered or expanded the ranges of many important arthropod vectors, along with their associated pathogens (e.g., dengue virus, West Nile virus, Trypanosoma cruzi [Chagas disease], Plasmodium sp.[malaria], etc.) (Kilpatrick $\&$ Randolph, 2012). Many bacterial diseases that have been well-controlled in developing countries by antimicrobial drugs are reemerging as resistance evolves simultaneously with more people using antimicrobials (Jones et al., 2008), such as drug-resistant staphylococcal or tuberculosis infections. Because of these pressing issues, it is keenly important for engineers and biological scientists to understand risk sciences to improve the design and selection of engineering controls and other mitigation strategies.

A ten-day long, yearly short course was developed to train engineers, biological and social scientists in QMRA approaches - the Quantitative Microbial Risk Assessment Interdisciplinary Instructional Institute (QMRAIII). Participants of QMRA III (1) attend lectures from the top scientists in the QMRA field; (2) engage in specific hands-on exercises; (3) under the mentorship of a program faculty member and teaching assistant, conduct a QMRA as a research case study within a multidisciplinary team; and (4) use and contribute to the QMRAwiki - an interactive, online platform.

The course covers the fundamental mathematical modeling techniques central to the four step QMRA paradigm:

i. Hazard identification - the quantitative description of the characteristics of microbial agents and hosts, their interactions, the disease process itself and the adverse consequences to the host from infection;

ii. Dose-response modeling - the development of biologically plausible, mechanistic models to describe the risk of infection, illness or mortality for a given exposure dose; 
iii. Exposure assessment - methods for microorganism detection in various environments including molecular tools; statistical and biophysical process models to describe occurrence, persistence, fate and transport, inactivation, excretion and secondary transmission of microbial agents;

iv. Risk characterization - simulation and statistical modeling to integrate the anticipated exposure dose from the exposure assessment with how much risk is associated with different doses from the dose-response model

Additionally, the course addresses social science topics related to risk assessment:

i. Risk Management - identification and evaluation of risk mitigation strategies, environmental engineering control measures or medical countermeasures using costbenefit, cost-effectiveness and decision analysis;

ii. Risk Communication- the theory and practice of exchanging information about risk among and between risk assessors, risk managers, and other interested parties; as well as understanding the perception of risk.

While the course was developed as a workshop over a short period of time, the contact hours are equivalent to that of a 3 credit hour course. Participants met in a classroom/computer lab for the majority of each day followed by small and large group dinners with other participants and faculty. Often groups met after dinner to work on the group projects as well.

The course followed established guidelines for the selection of teaching methodology to enhance learning (Felder et al 2000). The instructional objectives were defined and clearly articulated. The outcomes developed for the course are that upon completion of the course, students will:

1. Understand and be able to apply the quantitative skills and knowledge pertinent to constructing and using a QMRA

2. Understand and be able to apply the underlying concepts of QMRA

3. Understand the underlying concepts and techniques pertinent to developing a management and communication strategy related to QMRA

4. Develop an understanding of the wider world of QMRA and risk analysis

5. Build interdisciplinary collaborations

6. Increase use of QMRA framework

Specific objectives were developed for each outcome. For example, under the first outcome, students will be able to apply (1) microbiology applied to QMRA; (2) transport physics applied to QMRA; and (3) statistical and probabilistic skills and techniques applied to QMRA. The relevance of the course material was defined in the context of an existing or emerging health hazard requiring multidisciplinary solutions. Fate and transport modeling was defined by scenario like for waterborne pathogens resulting in high consequence outcomes through recreational exposures. Related scientific literature are provided for comparing student solutions with expert solutions for real world outbreaks. For each topic, abstract and concrete information is balanced. Most lectures are immediately followed up with an exercise requiring the student to use the material just covered immediately. Furthermore, the projects require application of course material in a short time frame and force participants to recall information from the lectures and exercises. Both active and cooperative learning techniques are utilized throughout the course. Exercises and problems are offered at the individual level and group level. The 
multidisciplinary groups are formed based on a preliminary survey of skills through the application process. The teams are designed to provide each group with collective complementary skills - a microbiologists, an engineer, an experienced data analyzer, etc. Teams are also asked to make progress presentations throughout the course on specific milestones and team functionality. Finally, a sense of concern for the students' learning is conveyed through personal interactions, over shared breaks and through mentoring. Each case study group has two faculty mentors assigned to them. The faculty mentors guide the team through the application and synthesis of new knowledge in order to produce a novel risk assessment by the end of the course.

During the first QMRA III Workshop, which was held from August 1 to 14, 2015, participants were evaluated on five Perceptions scales (Perceptions of: QMRA-Related Ability, QMRASpecific Ability, Knowledge - Hazard Identification, Knowledge-Dose Response and Exposure Assessment, Knowledge - Risk Management) through pre-and post- workshop evaluations. The pre-workshop evaluation of the QMRA III Workshop 2015 was conducted between June 29, and August 1, 2015. Post-workshop evaluations were completed between August 11 and August 16, 2015. Observations were also conducted three times during the workshop.

\section{Methods}

The workshop was evaluated through observation, Likert-type scale surveys, and open-ended prompts. Items were designed for this workshop - as such, validity and reliability of scales was established. All factor analyses used the maximum likelihood method.

In all, 24 participants completed the pre-survey and 26 completed the post-survey; data from 11 participants could be matched between the pre- and post-surveys. Participants completed identical pre- and post-workshop surveys containing five Perceptions scales: (Perceptions of: QMRA-Related Ability, QMRA-Specific Ability, Knowledge - Hazard Identification, Knowledge - Dose Response and Exposure Assessment, Knowledge - Risk Management). Given the small number of responses that could be matched, independent samples tests were run on the pre- and post-survey results.

Participants rated their ability in each of 11 questions regarding QMRA-related ability as No Ability, Low Ability, Intermediate Ability, or High Ability on. A score of 1 implies No Ability, while a score of 4 implies High Ability. Additionally, three ability items related to QMRAspecific abilities were also rated by the participants. These items did not align well with the more general abilities targeted in the QMRA-related Ability scale, and were evaluated independently. An average across items is used to calculate a scores for both Perceptions of QMRA-Related Ability and Perceptions of QMRA-Specific Ability. To evaluate knowledge in the three risk assessment paradigm areas (Hazard Identification, Dose Response and Exposure Assessment, an Risk Management), participants were asked to indicate the extent to which they understood specific concepts, with ratings of Strongly Disagree, Disagree, Agree, and Strongly Agree. A score of 1 implies low understanding (Strong Disagreement); a score of 4 implies high understanding (Strong Agreement). The number of items rated for Hazard Identification, Dose Response and Exposure Assessment, Risk Management were 11, 9, 5 respectively.

\section{Findings and Discussion}


Diversity among participants was achieved in the first cohort during the QMRAIII 2015 workshop. A total of 32 participants, 15 female and 17 male, attended the workshop. - . The selfidentified academic disciplines of the participants included engineering (Biosystems, Civil and/or Environmental) $(n=15)$; Public Health $(n=5)$; food science $(n=7)$; and microbiology (including environmental microbiology) $(n=6)$; and one other. Participants ranged in age from $26-56$, with an average age of $31.6 \pm 6.9$ years. Participants were $48 \%$ female $(n=11)$, with the remainder male $(n=12)$ and no transgendered participants. The population was about half Caucasian, with 52\% reporting exclusively Caucasian race/ethnicity $(n=11)$. Remaining participants, some of who fell into multiple ethnicity categories, were: Latino $(n=3)$; African/African American/Black ( $n=3)$; and Asian/Asian American $(n=4)$. Finally, the academic status of participants included graduate students $(n=10)$, M.S.-holding professionals $(n=5)$, and Ph.D.-holding professionals $(\mathrm{n}=6)$. Two additional participants classified themselves as "DVM holding professional" and "MPH".

Overall, participants experienced statistically significant increases in their perceptions of their ability and knowledge on all five scales as a result of instruction. (Table 1). The greatest achievements in meeting the learning objectives were observed in two categories of perception Perceptions of QMRA-Specific Ability and Perceptions of Knowledge - Risk Management. Within the QMRA-Specific Ability, students were asked to indicate their ability across the 4 steps in the QMRA paradigm along with other items related to modeling approached and software. As abilities in the core areas of QMRA (Figure 1) are the most vital to an implementable knowledge of QMRA, these results are highlighted.

Table 1. QMRAIII 2015 Pre and Post Workshop Scale Averages

\begin{tabular}{|l|c|c|}
\hline Scale & Pre & Post \\
\hline $\begin{array}{l}\text { Perceptions of QMRA-Related } \\
\text { Ability }\end{array}$ & $\begin{array}{c}1.80 \pm 0.57 \\
\text { (No to Low Ability) }\end{array}$ & $\begin{array}{c}2.67 \pm 0.51 \\
\text { (Low to Intermediate } \\
\text { Ability) }\end{array}$ \\
\hline $\begin{array}{l}\text { Perceptions of QMRA-Specific } \\
\text { Ability }\end{array}$ & $\begin{array}{c}2.03 \pm 0.80 \\
\text { (Low Ability) }\end{array}$ & $\begin{array}{c}3.47 \pm 0.62 \\
\text { (Intermediate to High } \\
\text { Ability) }\end{array}$ \\
\hline $\begin{array}{l}\text { Perceptions of Knowledge }- \\
\text { Hazard Identification }\end{array}$ & $\begin{array}{c}2.68 \pm 0.65 \\
\text { (Low to Intermediate } \\
\text { Understanding) }\end{array}$ & $\begin{array}{c}3.11 \pm 0.50 \\
\text { (Intermediate Understanding) }\end{array}$ \\
\hline $\begin{array}{l}\text { Perceptions of Knowledge }- \text { Dose } \\
\text { Response and Exposure }\end{array}$ & $\begin{array}{c}2.12 \pm 0.60 \\
\text { (Low Understanding) }\end{array}$ & $\begin{array}{c}3.24 \pm 0.65 \\
\text { (Intermediate Understanding) }\end{array}$ \\
\hline $\begin{array}{l}\text { Perceptions of Knowledge }- \text { Risk } \\
\text { Management }\end{array}$ & $\begin{array}{c}\text { 2.61 } \pm 0.64 \\
\text { (Low to Intermediate } \\
\text { Understanding) }\end{array}$ & $\begin{array}{c}3.34 \pm 0.42 \\
\text { (Intermediate to High } \\
\text { Understanding) }\end{array}$ \\
\hline
\end{tabular}




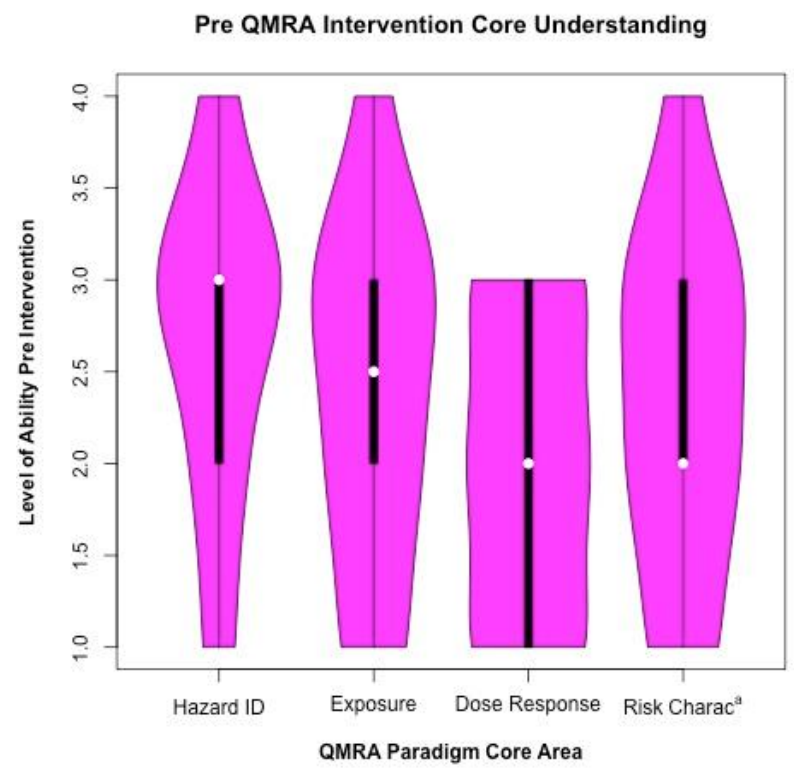

Figure 1. Violin plots for pre-intervention survey results

Hazard identification (Haz ID) is defined as the ability to prioritize and/or chose the appropriate pathogen based on specific criteria of interest. For the Haz ID the QMRA modeler can start from the symptomology or disease they desire to reduce, or can start from a target pathogen to reduce (e.g. from a drinking water regulatory sense). Therefore, for Haz ID microbiology and pathogenesis are the key fields in order to understand how to perform this task. Exposure Assessment is the portion of QMRA where the transport and survival of the pathogen is modeled to assess the likely dose the host will be exposed to. This is where physics, engineering and other heavily computational sciences shine in the QMRA. Dose response is the step in which the exposed dose is used to estimate a risk of infection, illness or death based on what the response being modeled is. Dose response models are most typically drawn from animal model experiments to allow for a more controlled dosing regimen. Finally risk characterization is taking the knowledge of uncertainty and variability modeling to incorporate these into the final risk assessment. For both of these steps, mathematics and statistics are the key fields of knowledge required. 
Table 2. Statistical Breakdown of Core Area Survey Items under Perceptions of QMRASpecific Ability

\begin{tabular}{|c|c|c|c|c|c|}
\hline & & Hazard ID & $\begin{array}{c}\text { Dose } \\
\text { Respons } \\
\text { e } \\
\end{array}$ & $\begin{array}{c}\text { Exposure } \\
\text { Assessment }\end{array}$ & $\begin{array}{c}\text { Risk } \\
\text { Characterizati } \\
\text { on }\end{array}$ \\
\hline \multirow{7}{*}{ 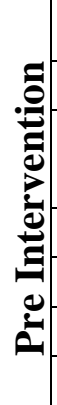 } & Median & 3.00 & 2.00 & 2.50 & 2.00 \\
\hline & Mean & 2.67 & 2.00 & 2.38 & 2.33 \\
\hline & $\begin{array}{l}\text { Standard } \\
\text { Deviation }\end{array}$ & 0.87 & 0.83 & 0.92 & 0.92 \\
\hline & Minimum & 1.00 & 1.00 & 1.00 & 1.00 \\
\hline & Maximum & 4.00 & 3.00 & 4.00 & 4.00 \\
\hline & Skewness & -0.56 & 0.00 & -0.14 & -0.01 \\
\hline & Kurtosis & -0.08 & -1.57 & -0.84 & -0.79 \\
\hline \multirow{7}{*}{ 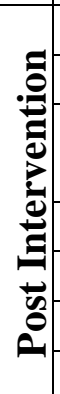 } & Median & 4.00 & 3.00 & 4.00 & 300 \\
\hline & Mean & 3.58 & 3.15 & 3.42 & 3.15 \\
\hline & $\begin{array}{l}\text { Standard } \\
\text { Deviation }\end{array}$ & 0.50 & 0.73 & 0.70 & 0.97 \\
\hline & Minimum & 3.00 & 1.00 & 2.00 & 2.00 \\
\hline & Maximum & 4.00 & 4.00 & 4.00 & 4.00 \\
\hline & Skewness & -0.33 & -0.92 & -0.83 & -1.48 \\
\hline & Kurtosis & -2.06 & 1.78 & -0.45 & 3.10 \\
\hline
\end{tabular}

When the students respond to questions about their abilities in each of these core areas, the structure of the core areas as described is not given to them. This is so as to not bias the results of the surveys. Rather the students are assumed to have some base level of knowledge in QMRA to have enrolled in the course. Table 2 shows the summary statistics from the pre- and post- 2015 QMRA III Summer Institute for the QMRA paradigm specific items. As can be seen, there was a relatively low perception of the participants' abilities ( 1 implies No Ability) for the pre- survey responses except for the Haz ID which can be perceived as qualitative or quantitative based on the disciplinary background. Considering the majority of the students in the 2015 class had significant microbiology knowledge or skills this is not a surprising result. Overall the rest of the core areas were scored in the middle ability (Low Ability; coded as 2). Examining the skew in the pre-intervention survey there is a left skew, but not overly large with a similar results on the kurtosis.

When examining Table 2 for the post-intervention survey we see a marked improvement in the median results from the 2015 students. Participants reported high ability in hazard identification and exposure assessment, followed by intermediate ability in dose-response and risk characterization. The skewness results were interesting in that for the Haz ID skew showed an improvement, however, this was not the case for the other core areas. From these initial results we can see that the course is likely designed well for a subset of the participants, but additional design elements should be included to improve the delivery of quantitative skills - dose-response modeling and exposure assessment. 
Violin plots in Figures 1 and 2 for pre- and post- intervention surveys respectively show a visual representation of the results. Violin plots are a mix of boxplots and kernel density plots giving a representation of the distribution of data. It is important to highlight and examine the density of the distribution of results from these surveys as we seek to capture the existence of bimodal distributions which may occur with cross-disciplinary training. Overall, we see again that there is a need to improve our computational instruction into QMRA for certain groups of participants.

Although there was an overall high response rate ( $n=24$ pre, $n=26$ post), because of limited paired data among the 2015 survey results $(n=11)$, combining this data with the 2016 survey results will allow us to determine if we are reaching particular subgroups and develop intervention strategies. It will allow to compare students from engineering disciplines with those from other fields across the key areas being evaluated.

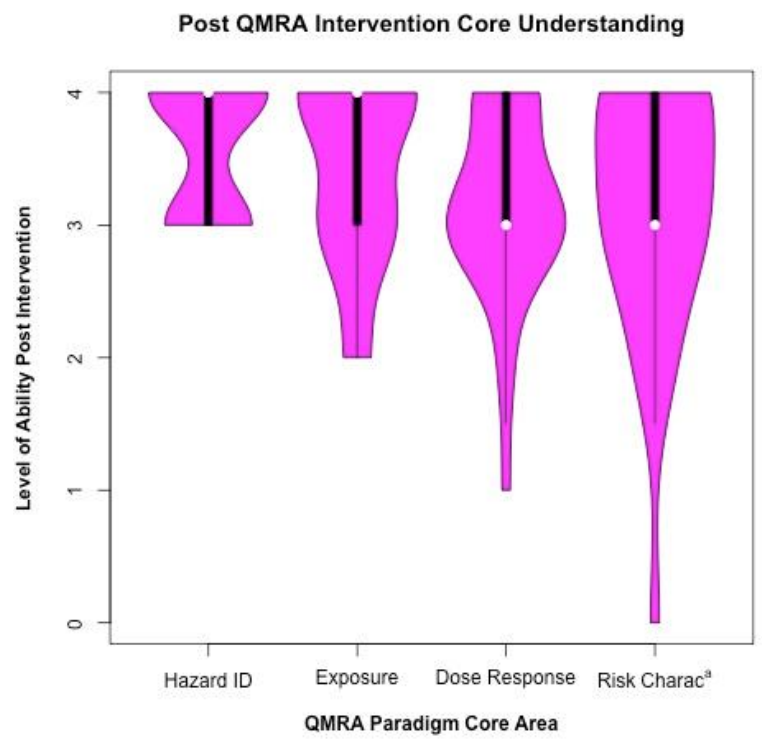

Figure 2. Violin plots for post-intervention survey results

\section{Conclusions}

Overall, the course evaluation presented herein indicates positive outcomes for participant learning. The course design is described and implements many previously published best practices for interdisciplinary and multidisciplinary education in the classroom - integration skills, teamwork, and communication (Borrego and Newswander, 2010, Faber et al 2014). The course also utilizes both active and cooperative learning techniques. Responses to open ended survey questions were also extremely positive. Examples are provided below:

- The workshop was an amazing, albeit grueling, experience. This really jump started my work in this field in a huge way! Thanks for allowing me to participate!

- Overall the course contents, professors, organization and quality was excellent.

- ...the workshop was very informative and gave a good starting point into the risk analysis world (I have no prior experience)

- A lot of us will feel empowered. 
Suggestions for the workshop included:

1. Reducing the amount of material covered in the workshop to allow participants more time to practice.

2. Provide more guidance about specific objectives of lessons and goals of activities

3. Make links between lectures and activities more obvious.

4. Reduce amount of lecture time and increase amount of time engaging with software/problems/activities.

5. Move lectures of first half of workshop and allow more time for activities and guidance during second half.

6. Put participants in explicit partnerships from the start of the workshop to facilitate peer-peer engagement

7. Make exercises easier to complete - for example, provide input variables so everyone gets the same output.

One additional common thread in the comments was a sense that people were overwhelmed by the material - some due to not being familiar with the topics and others from encountering new software/having problems with the software. This suggested that reducing the amount of material covered by the workshop and increasing time to engage in activities might be beneficial.

The feedback provided in 2015 was used to modify the design of the subsequent course in 2016. Pre-reading and recordings were prepared to reduce lecture time and orientate the group to the content prior to arrival. Lectures were reorganized so that the participants felt they had the information they needed to apply to their projects in a timelier manner. A full day of interaction between groups and mentors was accommodated in the schedule without lengthening the course. A common framework - the risk assessment paradigm - was introduced early as an organizing framework for the lectures and activities.

While the compressed schedule presents problems like "overwhelming" students, the timeframe for contact among student group members and pressure to complete a group project in 10 days abbreviated the team formation process and forced mutual reliance early as well. Compared to courses instructed over a 15 week semester, which the faculty regularly instruct at their respective universities, the short course reduces variability in the outcomes and team dynamic issues that generally arise.

The short course format and close contact between faculty and participants, who all traveled for the course, also fostered interaction between individuals from multiple disciplines. It also facilitated the sense of care and concern the program directors and resource faculty have for the growth of each participant, an interest in their future career goals and potential for future collaboration.

This is a work in progress and a 5 year project. Our future work will be to determine the success of changes made over time based on incorporation of feedback and best practices from the literature.

\section{Acknowledgements}

Funding for this research was provided by QRMA III, which is supported by NIGMS/NIH, Award Number R25GM108593. 


\section{References}

Borrego M and Newswander LK (2010) Definitions of interdisciplinary research: Toward graduate-level interdisciplinary learning outcomes. The Review of Higher Education, 34(1), 6184.

Faber MH, Giuliani L, Revez A, Jayasena S, Sparf J and Mendez JM (2014) Interdisciplinary approach to disaster resilience education and research. Procedia Economics and Finance, 18, 601-609.

Felder RM, Stice JE, and Rugarcia A (2000) The future of engineering education. VI. Making reform happen. Chemical Engineering Education, 34(3), 208-215.

Jones KE, Patel NG, Levy MA, Storeygard A, Balk D, Gittleman JL and Daszak P (2008) Global trends in emerging infectious diseases. Nature. 451 (7181), 990-3.

Kilpatrick AM and Randolph SE (2012) Drivers, dynamics, and control of emerging vectorborne zoonotic diseases. Lancet. 380 (9857), 1946-1955. 